\title{
ASSOCIATED NONCOMMUTATIVE VECTOR BUNDLES OVER THE VAKSMAN-SOIBELMAN QUANTUM COMPLEX PROJECTIVE SPACES
}

\author{
FRANCESCA ARICI \\ Mathematical Institute, Leiden University \\ P.O. Box 9512, 2300 RA Leiden, the Netherlands \\ ORCID:0000-0001-8326-6135_E-mail: f.arici@math.leidenuniv.nl \\ PIOTR M. HAJAC and MARIUSZ TOBOLSKI \\ Instytut Matematyczny Polskiej Akademii Nauk \\ Śniadeckich 8, 00-656 Warszawa, Poland \\ ORCID: 0000-0001-9764-5697, 0000-0001-7r38-8515 \\ E-mail:pmh@impan.pl,mtobolski@impan.pl
}

\begin{abstract}
By a diagonal embedding of $U(1)$ in $S U_{q}(m)$, we prolongate the diagonal circle action on the Vaksman-Soibelman quantum sphere $S_{q}^{2 n+1}$ to the $S U_{q}(m)$-action on the prolongated bundle. Then we prove that the noncommutative vector bundles associated via the fundamental representation of $S U_{q}(m)$, for $m \in\{2, \ldots, n\}$, yield generators of the even K-theory group of the $\mathrm{C}^{*}$-algebra of the Vaksman-Soibelman quantum complex projective space $\mathbb{C P}_{q}^{n}$.
\end{abstract}

1. Introduction. The K-theory of complex projective spaces was unraveled by Atiyah and Todd in [6, Propositions 2.3, 3.1 and 3.3] (cf. [1, Theorem 7.2] and [20, Corollary IV.2.8]). Denoting by $\mathrm{L}_{1}^{n}$ the dual tautological line bundle over $\mathbb{C P}^{n}$ and setting

$$
\mathrm{t}:=\left[\mathbb{C P}^{n} \times \mathbb{C}\right]-\left[\mathrm{L}_{1}^{n}\right] \in K^{0}\left(\mathbb{C P}^{n}\right),
$$

one obtains

$$
K^{0}\left(\mathbb{C P}^{n}\right)=\mathbb{Z}[\mathrm{t}] / \mathrm{t}^{n+1}
$$

2010 Mathematics Subject Classification: 46L80, 58B32.

Key words and phrases: K-theory, compact quantum groups, noncommutative bundles, PeterWeyl decomposition.

The paper is in final form and no version of it will be published elsewhere. 
Recently, this result was extended to the Vaksman-Soibelman quantum complex projective spaces [2, Propositions 3.3 and 3.4]:

$$
K_{0}\left(C\left(\mathbb{C P}_{q}^{n}\right)\right)=\mathbb{Z}[t] / t^{n+1} \cong \mathbb{Z}^{n+1} .
$$

Here $0<q<1$,

$$
t^{k}:=\sum_{m=0}^{k}(-1)^{m}\left(\begin{array}{c}
k \\
m
\end{array}\right)\left[\left(L_{1}^{n}\right)^{\otimes_{C\left(\mathbb{C P}_{q}^{n}\right)}^{m}}\right], 0 \leq k \leq n+1, \quad\left(L_{1}^{n}\right)^{\otimes_{C\left(\mathbb{C P}_{q}^{n}\right)}^{0}}:=C\left(\mathbb{C P}_{q}^{n}\right),
$$

and $L_{1}^{n}$ is the section bimodule of the noncommutative dual tautological line bundle over $\mathbb{C P}_{q}^{n}$.

The K-theory of both the Vaksman-Soibelman and the multipullback quantum complex projective planes [13, 23, 17] was thoroughly analysed in [11. In this special $n=2$ case, the elements

$$
[1], \quad\left[L_{1}^{2}\right]-[1], \quad\left[L_{-1}^{2} \oplus L_{1}^{2}\right]-2[1]
$$

form a basis of $K_{0}\left(C\left(\mathbb{C P}_{q}^{2}\right)\right)$. Here $L_{-1}^{2}$ is the section module of the noncommutative tautological line bundle over $\mathbb{C P}_{q}^{2}$.

A particular feature of the module $L_{-1}^{2} \oplus L_{1}^{2}$ established in [1] is that it can be obtained as a Milnor module [21, Theorem 2.1] constructed from the fundamental representation of $S U_{q}(2)$. This was achieved by taking $U(1)$ as a subgroup of $S U_{q}(2)$, and then realising $L_{-1}^{2} \oplus L_{1}^{2}$ as the section module of the noncommutative vector bundle associated to the prolongation $S_{q}^{5} \times_{U(1)} S U_{q}(2)$ via the fundamental representation of $S U_{q}(2)$. The upshot of having $\left[L_{-1}^{2} \oplus L_{1}^{2}\right]-2[1]$ as the image of a $\mathrm{C}^{*}$-algebraic Milnor connecting homomorphism [16. Section 0.4] is that it allows one to prove, without using the index pairing over $\mathbb{C P}_{q}^{2}$, that the set 1.4 is a basis of $K_{0}\left(C\left(\mathbb{C P}_{q}^{2}\right)\right)$.

The goal of this paper is to show that the above construction works in any dimension. More precisely, except for the classes $[1]$ and $\left[L_{1}^{n}\right]-[1]$, we prove that all other generators of $K_{0}\left(C\left(\mathbb{C P}_{q}^{n}\right)\right)$ come from section modules of noncommutative vector bundles associated to prolongations $S_{q}^{2 n+1} \times_{U(1)} S U_{q}(m)$ via the fundamental representations of $S U_{q}(m)$, respectively, where $m \in\{2, \ldots, n\}$.

We complement this way the Milnor vantage point on bases of $K_{0}\left(C\left(\mathbb{C P}_{q}^{n}\right)\right)$ coming from the exact sequence 3

$$
0 \longrightarrow K_{1}\left(C\left(S_{q}^{2 n-1}\right)\right) \stackrel{\partial_{10}}{\longrightarrow} K_{0}\left(C\left(\mathbb{C P}_{q}^{n}\right)\right) \longrightarrow K_{0}\left(C\left(\mathbb{C P}_{q}^{n-1}\right)\right) \longrightarrow 0 .
$$

Note that a generator of $K_{0}\left(C\left(\mathbb{C P}_{q}^{n}\right)\right)$ that does not come from $\mathbb{C P}_{q}^{n-1}$ can always be constructed via the Milnor connecting homomorphism $\partial_{10}$ from a generator of $K_{1}\left(C\left(S_{q}^{2 n-1}\right)\right)$. Therefore, by iteration, we can obtain a basis of $K_{0}\left(C\left(\mathbb{C P}_{q}^{n}\right)\right)$ from the Milnor connecting homomorphisms.

Finally, as $S_{q}^{2 n-1}$ is a homogeneous space of $S U_{q}(n)$, we arrive at the following: Question. Can a generator of $K_{1}\left(C\left(S_{q}^{2 n-1}\right)\right) \cong \mathbb{Z}$ be always expressed in terms of representations of $S U_{q}(n)$ ?

This question has positive answer for $q=1$ by the work of Harris [18] based on the work of Hodgkin and Atiyah [19, 5]. For $0<q<1$ and $n=2$, we just take the fundamental representation of $S U_{q}(2)$. 
2. Preliminaries. The Vaksman-Soibelman odd quantum spheres [26] are defined as quantum homogeneous spaces for Woronowicz's quantum special unitary groups [27]:

$$
C\left(S_{q}^{2 n+1}\right):=C\left(S U_{q}(n+1)\right)^{S U_{q}(n)} .
$$

Here $0<q \leq 1$, and we use the notation $A^{\mathbb{G}}$ for the fixed-point subalgebra of a $\mathrm{C}^{*}$-algebra $A$ under an action of a compact quantum group $\mathbb{G}$. (Note that the $q=1$ case recovers the classical situation.) One can show that $C\left(S_{q}^{2 n+1}\right)$ is the universal $\mathrm{C}^{*}$-algebra given by the following generators and relations:

$$
\begin{gathered}
z_{i} z_{j}=q z_{j} z_{i} \quad \text { for } i<j, \quad z_{i} z_{j}^{*}=q z_{j}^{*} z_{i} \quad \text { for } i \neq j, \\
z_{i} z_{i}^{*}=z_{i}^{*} z_{i}+\left(q^{-2}-1\right) \sum_{m=i+1}^{n} z_{m} z_{m}^{*}, \quad \sum_{m=0}^{n} z_{m} z_{m}^{*}=1 .
\end{gathered}
$$

Much like in the classical case, the Vaksman-Soibelman quantum odd spheres enjoy the diagonal circle action given on generators by

$$
\left(z_{0}, z_{1}, \ldots, z_{n}\right) \mapsto\left(\lambda z_{0}, \lambda z_{1}, \ldots, \lambda z_{n}\right), \quad \lambda \in U(1) .
$$

One uses this action to define the quantum complex projective spaces [26]

$$
C\left(\mathbb{C P}_{q}^{n}\right):=C\left(S_{q}^{2 n+1}\right)^{U(1)} .
$$

Recall that freeness of circle actions can be characterised in terms of their spectral subspaces (e.g., see [22]). Given an action $\alpha: U(1) \rightarrow \operatorname{Aut}(A)$ on a unital $\mathrm{C}^{*}$-algebra, for each character $m \in \mathbb{Z}$, one defines the $m$-th spectral subspace $A_{m}$ as

$$
A_{m}:=\left\{a \in A \mid \alpha_{\lambda}(a)=\lambda^{m} a \text { for all } \lambda \in U(1)\right\} .
$$

The subspace $A_{0}$ agrees with the fixed-point subalgebra $A^{U(1)}$, and the inclusions $A_{m} A_{n} \subseteq A_{m+n}, m, n \in \mathbb{Z}$, turn $A$ into a $\mathbb{Z}$-graded algebra. Now, one can say that the action $\alpha$ is free if and only if the $\mathbb{Z}$-grading is strong [25], i.e. $A_{m} A_{n}=A_{m+n}$ for all $m, n \in \mathbb{Z}$. It is straightforward to check that the bimodules $A_{m}$ are finitely generated projective both as left and right $A^{U(1)}$-modules. Furthermore, they are invertible and they can be interpreted as modules of sections of associated noncommutative line bundles (e.g., see [4]).

Note that, using the spatial tensor product, the action $\alpha$ can be dualised to the coaction

$$
\delta: A \longrightarrow A \underset{\min }{\otimes} C(U(1))=C(U(1), A), \quad \delta(a)(\lambda):=\alpha_{\lambda}(a) .
$$

Denote by $\mathcal{O}(U(1))$ the dense Hopf subalgebra of $C(U(1))$ consisting of Laurent polynomials in one variable. The Peter-Weyl $\mathcal{O}(U(1))$-comodule algebra $\mathcal{P}_{U(1)}(A)$, defined as the set of all $a \in A$ such that $\delta(a) \in A \otimes \mathcal{O}(U(1))$ (see [7]), is the purely algebraic direct sum of spectral subspaces:

$$
\mathcal{P}_{U(1)}(A)=\bigoplus_{m \in \mathbb{Z}} A_{m} .
$$

The $\mathcal{P}_{U(1)}(A)$ comodule algebra is principal in the sense of [14].

A centrepiece concept for our paper is the notion of a prolongation of a principal comodule algebra. To define it, first we need to recall the definition of the cotensor product. For a coalgebra $C$, the cotensor product of a right $C$-comodule $M$ with a coaction 
$\rho_{R}: M \rightarrow M \otimes C$ and a left $C$-comodule $N$ with a coaction $\rho_{L}: N \rightarrow C \otimes N$ is defined as the difference kernel

$$
M \stackrel{C}{\square} N:=\operatorname{ker}\left(\rho_{R} \otimes \mathrm{id}-\mathrm{id} \otimes \rho_{L}: M \otimes N \longrightarrow M \otimes C \otimes N\right) .
$$

Given a surjection of Hopf algebras $\pi: \mathcal{H} \rightarrow \overline{\mathcal{H}}$, we can treat $\mathcal{H}$ as a left $\overline{\mathcal{H}}$-comodule via the coaction $(\pi \otimes \mathrm{id}) \circ \Delta$. For any right $\overline{\mathcal{H}}$-comodule algebra $\mathcal{P}$, we define its prolongation as the cotensor product $\mathcal{P} \square^{\overline{\mathcal{H}}} \mathcal{H}$. It is easy to check that this cotensor product is a right $\mathcal{H}$-comodule algebra for the coaction id $\otimes \Delta$. It is also straightforward to verify that, if $\mathcal{P}$ is principal, then so is its prolongation.

Let $\mathcal{P}$ be a principal $\mathcal{H}$-comodule algebra with a coaction $\Delta_{R}$ and let $V$ be a left $\mathcal{H}$-comodule. Much as in the classical case, we can form the associated left module $\mathcal{P} \square^{\mathcal{H}} V$ over the coaction-invariant subalgebra $\mathcal{P}^{\text {co } \mathcal{H}}:=\left\{a \in \mathcal{P} \mid \Delta_{R}(a)=a \otimes 1\right\}$. We think of this module as the section module of the associated noncommutative vector bundle. If $\mathcal{P}$ is principal and $V$ is finite dimensional, then it is known that the associated module is finitely generated projective (e.g., see [8, Theorem 3.1]).

3. Line bundles. As explained in the introduction, the K-theory of the VaksmanSoibelman quantum complex projective spaces is determined by section modules of associated noncommutative line bundles. Therefore, we begin our considerations by putting together some facts involving these noncommutative line bundles.

For the Vaksman-Soibelman quantum sphere $S_{q}^{2 n+1}$, the Peter-Weyl subalgebra (2.1) becomes

$$
\mathcal{P}_{U(1)}\left(C\left(S_{q}^{2 n+1}\right)\right)=\bigoplus_{m \in \mathbb{Z}} L_{m}^{n}
$$

where

$$
L_{m}^{n}:=\left\{a \in C\left(S_{q}^{2 n+1}\right) \mid \alpha_{\lambda}(a)=\lambda^{m} a \text { for all } \lambda \in U(1)\right\} \cong \begin{cases}\left(L_{1}^{n}\right)^{\otimes_{C\left(\mathrm{CP}_{q}^{n}\right)}^{m}} & \text { for } m>0 \\ C\left(\mathbb{C P}_{q}^{n}\right) & \text { for } m=0 \\ \left(L_{-1}^{n}\right)^{\otimes_{C\left(\mathbb{C P} q_{q}^{n}\right)}} & \text { for } m<0 .\end{cases}
$$

The $U(1)$-action on $C\left(S_{q}^{2 n+1}\right)$ is free by [24, Corollary 3], so we can think of $L_{m}^{n}$ as modules of sections of associated noncommutative line bundles. Note that our sign convention is opposite to that used in [2], i.e. $L_{m}^{n}=\mathcal{L}_{-m}$.

Recall that there exists a $U(1)$-equivariant $*$-homomorphism $\varphi: C\left(S_{q}^{2 n+1}\right) \rightarrow C\left(S_{q}^{3}\right)$ given on standard generators by

$$
\varphi\left(z_{i}\right)= \begin{cases}z_{0} & \text { if } i=0 \\ z_{1} & \text { if } i=1 \\ 0 & \text { if } i \geq 2\end{cases}
$$

Therefore, by [15, Theorem 0.1], the induced map

$$
\left(\left.\varphi\right|_{C\left(\mathbb{C P}_{q}^{n}\right)}\right)_{*}: K_{0}\left(C\left(\mathbb{C P}_{q}^{n}\right)\right) \longrightarrow K_{0}\left(C\left(\mathbb{C P}_{q}^{1}\right)\right)
$$

satisfies

$$
\left(\left.\varphi\right|_{C\left(\mathbb{C P}_{q}^{n}\right)}\right)_{*}\left(\left[L_{m}^{n}\right]\right)=\left[L_{m}^{1}\right]
$$


for any $m \in \mathbb{Z}$. Since the index pairing computation of [12, Theorem 2.1] proves that $\left[L_{m}^{1}\right]=\left[L_{k}^{1}\right]$ implies $m=k$ for $0<q<1$, and the $q=1$ case is well known, we thus arrive at the following:

THEOREM 3.1. The spectral subspaces $L_{m}^{n}$ are pairwise stably non-isomorphic as finitely generated projective left modules over $C\left(\mathbb{C P}_{q}^{n}\right), 0<q \leq 1$. That is, for all $n \in \mathbb{N} \backslash\{0\}$ and any $m, k \in \mathbb{Z}$, we have

$$
\left[L_{m}^{n}\right]=\left[L_{k}^{n}\right] \quad \text { if and only if } m=k .
$$

Observe that, for $0<q<1$, this fact was already proven in [10, Propositions 4 and 5] using the index pairing between the K-homology group $K^{0}\left(C\left(\mathbb{C P}_{q}^{n}\right)\right)$ and the K-theory group $K_{0}\left(C\left(\mathbb{C P}_{q}^{n}\right)\right)$.

To use the index pairing, for the rest of this section we assume $0<q<1$. Denote by $\left[\mu_{0}\right], \ldots,\left[\mu_{n}\right] \in K^{0}\left(C\left(\mathbb{C P}_{q}^{n}\right)\right)$ the K-homology generators constructed in [10, Section 2]. In the same paper, the authors proved that

$$
\left\langle\left[\mu_{k}\right],\left[L_{m}^{n}\right]\right\rangle=\left(\begin{array}{c}
m \\
k
\end{array}\right)
$$

for all $m \in \mathbb{N}$ and for all $0 \leq k \leq n$. Here we adopt the convention that $\left(\begin{array}{c}m \\ k\end{array}\right):=0$ when $k>m$. In particular,

$$
\left\langle\left[\mu_{0}\right],\left[L_{m}^{n}\right]\right\rangle=1 \quad \text { and }\left\langle\left[\mu_{1}\right],\left[L_{m}^{n}\right]\right\rangle=m
$$

for all $m \in \mathbb{Z}$ (see 2, Proposition 3.2]). We view the pairing with $\left[\mu_{0}\right]$ as computing the rank, and the pairing with $\left[\mu_{1}\right]$ as computing the noncommutative first Chern class. In agreement with the classical setting, we call $L_{-1}^{n}$ the section module of the noncommutative tautological line bundle, and we refer to $L_{1}^{n}$ as the section module of the noncommutative dual tautological line bundle. The latter is also known as the noncommutative Hopf line bundle.

As mentioned in the introduction, the K-theory groups of quantum projective spaces have the same bases as their classical counterparts. This was proven in [2] by showing that, for $0 \leq j \leq n$ and for $0 \leq k \leq n$,

$$
\left\langle\left[\mu_{k}\right], t^{j}\right\rangle=\left\{\begin{array}{lll}
0 & \text { for } & j \neq k \\
(-1)^{j} & \text { for } & j=k
\end{array}\right.
$$

Recall that $t:=[1]-\left[L_{1}^{n}\right]$ is the noncommutative Euler class of $L_{1}^{n}$ (see 1.3$)$ ).

We conclude this section by computing the pairings of the K-homology generators with the class of the section module of the noncommutative tautological line bundle $L_{-1}^{n}$. Proposition 3.2. For all positive integers $n$ and for all $0 \leq k \leq n$, we have

$$
\left\langle\left[\mu_{k}\right],\left[L_{-1}^{n}\right]\right\rangle=(-1)^{k} .
$$

Proof. The cases $k=0,1$ are covered by 3.2 . For $2 \leq k \leq n$, we infer from [9] the identity

$$
\left[L_{-1}^{n}\right]=1+t+\ldots+t^{n}
$$

and combine it with the pairings in 3.3 . 
4. Vector bundles. Although the $K_{0}$-groups of the Vaksman-Soibelman quantum projective spaces are determined by associated noncommutative line bundles, expressing the generators as associated noncommutative vector bundles has its merits, as explained in the introduction.

For every $0<q \leq 1$ and $m=2, \ldots, n$, consider the following surjection of Hopf algebras

$$
\pi_{m}: \mathcal{O}\left(S U_{q}(m)\right) \longrightarrow \mathcal{O}(U(1)), \quad \pi_{m}\left(U_{i j}\right):= \begin{cases}\delta_{i j} u^{-1} & \text { for } i<n \\ \delta_{i j} u^{m-1} & \text { for } i=n,\end{cases}
$$

where $U_{i j}$ are the matrix coefficients of the fundamental representation of $S U_{q}(m)$. To prove that $\pi_{m}$ is well defined, we have to verify that the determinant formulae (1.17) and (1.18) in [27] are satisfied. This can be done by a direct computation taking advantage of the fact that $u$ is unitary and that $\pi_{m}$ assigns zero to off-diagonal entries. With the help of $\pi_{m}$, we define the prolongations of principal comodule algebras:

$$
\mathcal{P}_{U(1)}\left(C\left(S_{q}^{2 n+1}\right)\right) \stackrel{\mathcal{O}(U(1))}{\square} \mathcal{O}\left(S U_{q}(m)\right) .
$$

Next, taking the fundamental representation $V_{m}$ of $S U_{q}(m)$, we construct the associated finitely generated projective left $C\left(\mathbb{C P}_{q}^{n}\right)$-modules

$$
\mathcal{F}_{m}^{n}:=\mathcal{P}_{U(1)}\left(C\left(S_{q}^{2 n+1}\right)\right) \stackrel{\mathcal{O}(U(1))}{\square} \mathcal{O}\left(S U_{q}(m)\right) \stackrel{\mathcal{O}\left(S U_{q}(m)\right)}{\square} V_{m}
$$

We are now ready to prove our main result:

THEOREM 4.1. For any positive integer $n$ and $0<q \leq 1$, the classes

$$
\mathcal{E}_{0}^{n}:=[1], \quad \mathcal{E}_{1}^{n}:=\left[L_{1}^{n}\right]-[1], \quad \mathcal{E}_{m}^{n}:=\left[\mathcal{F}_{m}^{n}\right]-m[1], \quad m=2, \ldots, n,
$$

form a basis of $K_{0}\left(C\left(\mathbb{C P}_{q}^{n}\right)\right)$.

Proof. To begin with, plugging in the explicit formula (4.1) into the definition (4.2), we derive the decomposition

$$
\mathcal{F}_{m}^{n}=\left(L_{-1}^{n}\right)^{\oplus(m-1)} \oplus L_{m-1}^{n} .
$$

Combining (1.1) and 1.2 , we know that $\left\{1, t, \ldots, t^{n}\right\}$ is a basis of $K_{0}\left(C\left(\mathbb{C P}_{q}^{n}\right)\right)$. We now use (3.4) to write $\mathcal{E}_{m}^{n}$ in the above basis

$$
\mathcal{E}_{0}^{n}=1, \quad \mathcal{E}_{1}^{n}=-t, \quad \mathcal{E}_{m}^{n}=\sum_{k=2}^{n}\left((m-1)+(-1)^{k}\left(\begin{array}{c}
m-1 \\
k
\end{array}\right)\right) t^{k}, \quad m \geq 2 .
$$

It remains to show that the matrix $\mathrm{M}_{n}$ implementing 4.3 is invertible over the integers. Since $\mathrm{M}_{n+1}$ always contains $\mathrm{M}_{n}$ as an $n \times n$ submatrix in the upper-left corner, it is straightforward to verify the claim using elementary row operations and induction.

For $n=2$, the above theorem yields

$$
[1], \quad\left[L_{1}^{2}\right]-[1], \quad\left[L_{-1}^{2} \oplus L_{1}^{2}\right]-2[1]
$$

as a basis of $K_{0}\left(C\left(\mathbb{C P}_{q}^{2}\right)\right)$. This agrees with the $\mathrm{K}$-theory computations done in [11 for the multipullback quantum complex projective plane. Note also that the matrix $\mathrm{M}_{n}$ used in the above proof agrees up to a sign with the matrix of pairings $\left\langle\left[\mu_{k}\right], \mathcal{E}_{j}^{n}\right\rangle$, which can be computed using (3.1) and Proposition 3.2 
Acknowledgements. This work is part of the project Quantum Dynamics partially supported by EU-grant H2020-MSCA-RISE-2015-691246 and Polish Government grants 3542/H2020/2016/2 and 328941/PnH/2016. We acknowledge a substantial logistic support from the Max Planck Institute for Mathematics in the Sciences in Leipzig, where much of this research was carried out. It is a pleasure to thank Nigel Higson for discussions and references.

\section{References}

[1] J. F. Adams, Vector fields on spheres, Ann. of Math. (2) 75 (1962), 603-632.

[2] F. Arici, S. Brain, G. Landi, The Gysin sequence for quantum lens spaces, J. Noncommut. Geom. 9 (2015), 1077-1111.

[3] F. Arici, F. D'Andrea, P. M. Hajac, M. Tobolski, An equivariant pullback structure of trimmable graph $C^{*}$-algebras, J. Noncommut. Geom., to appear, arXiv:1712.08010.

[4] F. Arici, J. Kaad, G. Landi, Pimsner algebras and Gysin sequences from principal circle actions, J. Noncommut. Geom. 10 (2016), 29-64.

[5] M. F. Atiyah, On the K-theory of compact Lie groups, Topology 4 (1965), 95-99.

[6] M. F. Atiyah, J. A. Todd, On complex Stiefel manifolds, Proc. Cambridge Philos. Soc. 56 (1960), 342-353.

[7] P. F. Baum, K. De Commer, P. M. Hajac, Free actions of compact quantum groups on unital $C^{*}$-algebras, Doc. Math. 22 (2017), 825-849.

[8] T. Brzeziński, P. M. Hajac, The Chern-Galois character, C. R. Math. Acad. Sci. Paris 338 (2004), 113-116.

[9] F. D'Andrea, P. M. Hajac, T. Maszczyk, A. Sheu, B. Zieliński, The K-theory type of quantized $C W$-complexes, arXiv:2002.09015.

[10] F. D'Andrea, G. Landi, Bounded and unbounded Fredholm modules for quantum projective spaces, J. K-Theory 6 (2010), 231-240.

[11] C. Farsi, P. M. Hajac, T. Maszczyk, B. Zieliński, Rank-two Milnor idempotents for the multipullback quantum complex projective plane, arXiv:1512.08816.

[12] P. M. Hajac, Bundles over quantum sphere and noncommutative index theorem, K-Theory 21 (2000), 141-150.

[13] P. M. Hajac, A. Kaygun, B. Zieliński, Quantum complex projective spaces from Toeplitz cubes, J. Noncommut. Geom. 6 (2012), 603-621.

[14] P. M. Hajac, U. Krähmer, R. Matthes, B. Zieliński, Piecewise principal comodule algebras, J. Noncommut. Geom. 5 (2011), 591-614.

[15] P. M. Hajac, T. Maszczyk, Pullbacks and nontriviality of associated noncommutative vector bundles, J. Noncommut. Geom. 12 (2018), 1341-1358.

[16] P. M. Hajac, A. Rennie, B. Zieliński, The K-theory of Heegaard quantum lens spaces, J. Noncommut. Geom. 7 (2013), 1185-1216.

[17] P. M. Hajac, J. Rudnik, Noncommutative bundles over the multi-pullback quantum complex projective plane, New York J. Math. 23 (2017), 295-313.

[18] B. Harris, The K-theory of a class of homogeneous spaces, Trans. Amer. Math. Soc. 131 (1968), 323-332.

[19] L. Hodgkin, On the K-theory of Lie groups, Topology 6 (1967), 1-36.

[20] M. Karoubi, K-Theory: an Introduction, Grundlehren Math. Wiss. 226, Springer, Berlin, 1978. 
[21] J. Milnor, Introduction to Algebraic K-Theory, Ann. of Math. Stud. 72, Princeton Univ. Press, Princeton, NJ, 1971.

[22] W. L. Paschke, K-Theory for actions of the circle group on $C^{*}$-algebras, J. Operator Theory 6 (1981), 125-133.

[23] J. Rudnik, The K-theory of the triple-Toeplitz deformation of the complex projective plane, in: Operator Theory and Quantum Groups, Banach Center Publ. 98, Polish Acad. Sci. Inst. Math., Warsaw, 2012, 303-310.

[24] W. Szymański, Quantum lens spaces and principal actions on graph $C^{*}$-algebras, in: Noncommutative Geometry and Quantum Groups, Banach Center Publ. 61, Polish Acad. Sci. Inst. Math., Warsaw, 2003, 299-304.

[25] K. H. Ulbrich, Vollgraduierte Algebren, Abh. Math. Sem. Univ. Hamburg 51 (1981), 136-148.

[26] L. L. Vaksman, Ya. S. Soǔbel'man, Algebra of functions on the quantum group $\mathrm{SU}(\mathrm{n}+1)$ and odd-dimensional quantum spheres, Algebra i Analiz 2 (1990), no. 5, 101-120; English transl.: Leningrad Math. J. 2 (1991), 1023-1042.

[27] S. L. Woronowicz, Tannaka-Krein duality for compact matrix pseudogroups. Twisted $\mathrm{SU}(N)$ group, Invent. Math. 93 (1988), 35-76. 